\title{
Encaminhamento Seletivo de Interesses em Redes Veiculares de Dados Nomeados Baseado no Tempo de Vida do Enlace
}

\author{
Antonio M. de Sousa ${ }^{1}$, Francisco R. C. Araújo ${ }^{1}$, Leobino N. Sampaio ${ }^{1}$ \\ ${ }^{1}$ Programa de Pós-Graduação em Ciência da Computação (PGCOMP) \\ Departamento de Ciência da Computação - Universidade Federal da Bahia (UFBA) \\ Salvador - BA - Brasil \\ \{antonio.mateus, franciscorca, leobino\}@ufba.br
}

\begin{abstract}
In recent years, the Vehicular Named Data Network (VNDN) architecture has been proposed to face performance and reliability challenges from IP/host centric Vehicular Ad hoc Networks. The constant mobility of nodes in such networks can result in rapid flooding of unsatisfied interest packets thus leading to network overloads. In this article, is proposed a link stability-based interest forwarding strategy that rely on predictions of link durations to reduce the number of retransmissions. The simulation results shows that the proposed strategy reduces the number of interests and data packets retransmissions while preserving the interests satisfaction rate.
\end{abstract}

Resumo. Recentemente, as Redes Veiculares de Dados Nomeados foram propostas para lidar com os desafios enfrentados pelas redes veiculares convencionais, que utilizam um esquema de endereçamento de nós baseado em IP. A mobilidade constante e imprevisível dos nós nessas redes causam inundação de pacotes de interesse não satisfeitos, que sobrecarregam a rede. Neste artigo é proposta uma estratégia de encaminhamento de interesses que visa reduzir o número de retransmissões ao utilizar o tempo de vida do enlace como critério de decisão no encaminhamento. Os resultados de simulação mostram que a estratégia proposta consegue reduzir o número de retransmissões de interesses e de dados enquanto mantém a taxa de satisfação de interesses estável.

\section{Introdução}

As redes veiculares de dados nomeados (do inglês, Vehicular Named Data Networks VNDN) são definidas como redes veiculares Ad hoc (Vehicular Ad hoc Networks - VANETs) em que os nós (veículos) atuam no armazenamento temporário de conteúdos e encaminhamento de interesses para nós adjacentes, no intuito de atender a demanda dos consumidores. Trata-se de um tipo de VANET que aproveita as propriedades das redes de dados nomeados (do inglês, Named Data Networking - NDN) [Saxena et al. 2016] para contornar as limitações relacionadas ao uso de endereços IPs no processo de descoberta e consumo de serviços [Ahmed et al. 2016b]. Arquiteturas NDNs possuem suporte nativo a mobilidade, comunicação não orientada à conexão e caching na camada de rede [Saxena et al. 2016]. Através de tais características, as VNDNs conseguem lidar com os desafios inerentes ao ambiente de VANETs, marcado pela imprevisibilidade e complexidade de topologias devido à constante mobilidade dos veículos.

Por herdar as propriedades da arquitetura NDN, a comunicação em VNDNs se baseia na utilização de pacotes de interesse e de dados para requisitar e obter conteúdos, 
respectivamente. Além disso, os nós utilizam as principais estruturas de dados utilizadas na comunicação em NDN, a saber: tabela de interesses pendentes (do inglês, Pending Interest Table - PIT) e base de conteúdos (do inglês, Content Store - CS). A PIT é responsável por armazenar a lista de interesses pendentes e interesses já satisfeitos, para detectar loops e coletar métricas. A CS consiste em uma cache que armazena os conteúdos disponíveis pelo nó e os conteúdos recebidos de outros nós, ao satisfazer um interesse [Yaqub et al. 2016]. Apesar da Forwarding Information Base (FIB) fazer parte da arquitetura NDN, a mesma não é utilizada em VNDN, pois a manutenção seria muito complexa devido a dinamicidade do meio e conteúdos de disponibilidade temporária [Karagiannis et al. 2011].

Dado que VNDN não utiliza FIBs no processo de roteamento, a principal abordagem adotada na descoberta de conteúdos consiste no envio de pacotes de interesses por difusão (broadcast). Esse modo de comunicação, contudo, resulta no problema de broadcast storm [Ahmed et al. 2015], em que os nós retransmitem sucessivas vezes o mesmo pacote para seus vizinhos até que o nó produtor do conteúdo seja alcançado ou seu tempo de vida expire [Grassi et al. 2015, Majeed et al. 2017]. Esse comportamento resulta em diversos subproblemas, tais como a saturação nos enlaces, interferências, lentidão, perdas, dentre outros [Kuai et al. 2016, Yu et al. 2013]. Por tais motivos, o problema de broadcast storm [Ahmed et al. 2015] de pacotes de interesse e pacotes de dados em VNDN passa a ser uma questão em aberto e que carece de maiores investigações.

Diferentes estratégias descritas na literatura têm sido adotadas para resolver o problema de broadcast storm pacotes em VNDN [Maryam et al. 2017, Ahmed et al. 2015, Ahmed et al. 2016b, Ahmed et al. 2016a]. Contudo, a maioria dessas buscam mitigar o efeito do problema por meio do encaminhamento via multicast. Para isso, tais estratégias trazem uma complexidade adicional à arquitetura NDN, incluindo novos campos nos pacotes e novas mensagens de controle. Visando superar tais problemas, este artigo apresenta a estratégia EVEN (Estratégia de encaminhamento seletivo de interesses em redes VEiculares de dados Nomeados). A EVEN promove um encaminhamento seletivo de interesses em VNDN com o objetivo de reduzir o número de retransmissões desnecessárias de pacotes de interesse com mínima modificação na arquitetura NDN padrão. Neste sentido, a estratégia considera como principal critério de encaminhamento o tempo de vida estimado do enlace entre os nós. Através dessa estimativa, os nós decidem se estão aptos a realizarem o encaminhamento do interesse recebido. Como resultado, apenas um subconjunto de nós são envolvidos na busca por conteúdos, reduzindo assim a ocorrência de broadcast storm de interesses na rede. Por conta de tais características, este trabalho apresenta as seguintes contribuições: i) uma nova estratégia que faz o encaminhamento seletivo de interesses em VNDN; ii) redução de broadcast storm com um mínimo de alterações na arquitetura NDN padrão.

A eficiência da estratégia EVEN foi verificada a partir de uma avaliação experimental feita por meio de simulação no ndnSim. O estudo avaliou as vantagens da proposta nos cenários urbano e de rodovia a partir da variação no número de veículos. Os resultados obtidos comprovaram que a EVEN consegue reduzir, significativamente, o número de retransmissões de interesses, em torno de $50 \%$ no cenário de rodovia e $34 \%$ no cenário urbano, e de dados, em $30 \%$ na rodovia e $34 \%$ no ambiente urbano, enquanto reduz o atraso e mantém uma razoável taxa de satisfação de interesses. 
Este trabalho está organizado da seguinte forma: a Seção 2 apresenta os trabalhos relacionados e a proposta é detalhada na Seção 3. Os experimentos são discutidos na Seção 4 e os resultados e avaliação de desempenho são mostrados na Seção 5. Por fim, as conclusões e trabalhos futuros estão presentes na Seção 6.

\section{Trabalhos Relacionados}

Em [Ahmed et al. 2015], os autores propuseram uma estratégia de encaminhamento que utiliza um método de tomada de decisão baseado em multi critério, no qual o consumidor/encaminhador pode selecionar apenas um veículo entre os seus vizinhos para encaminhar o interesse. A decisão de encaminhamento é realizada com o auxílio de duas novas estruturas de dados que cada veículo armazena localmente. A primeira estrutura consiste em uma lista de interesses satisfeitos pelos seus vizinhos chamada Neighbors Satisfied List (NSL) e uma lista chamada Recent Satisfied List (RSL) que armazena os interesses satisfeitos pelo próprio veículo. A RSL é trocada periodicamente entre vizinhos de um salto possibilitando, assim, escolher um nó para ser um encaminhador. Após determinar qual nó será o encaminhador, os demais vizinhos irão ignorar os pacotes de interesse que chegam.

Outra estratégia de encaminhamento em VNDN é o Navigo proposto em [Grassi et al. 2015], no qual os autores optaram por utilizar uma estratégia de encaminhamento baseado em geolocalização, que depende da presença de unidades de acostamento (do inglês, Road Side Units - RSUs) nas proximidades das vias e da cache dos veículos, onde um algoritmo direciona os interesses aos produtores de conteúdo utilizando o menor caminho entre os mesmos. O algoritmo funciona da seguinte forma: inicialmente, é realizado um mapeamento dos nomes dos dados para as suas localizações, em seguida, para manter as informações atualizadas os autores desenvolveram um mecanismo de descoberta e escolha de produtores. Como a topologia de VANETs muda constantemente é necessário sempre atualizar as rotas o que acarreta em uma sobrecarga computacional adicional.

Já em [Ahmed et al. 2016a], foi proposto um esquema para controlar a retransmissão de pacotes de dados em VNDN. O esquema consiste na adição de um campo no pacote de interesse para armazenar o hop count, ou quantidade de saltos realizados pelo pacote que ao chegar no produtor esta informação é copiada para o pacote de dados, que por sua vez é encaminhado ao consumidor. À medida que o pacote faz o caminho inverso até o consumidor, cada nó intermediário verifica se o hop count é menor ou igual ao TTL (Time To Live) se for verdade o nó decrementa o TTL e encaminha o pacote, caso contrário, o descarta.

A maioria das propostas citadas tentam prevenir o broadcast storm por meio de estratégias de encaminhamento multicast, ou seja, envia os interesses apenas para um subconjunto de nós intermediários julgados como aptos a executar a tarefa de encaminhamento. Apesar dos benefícios alcançados, as mesmas trazem complexidade adicional à arquitetura de NDN atual, adicionando novas estruturas de dados que acarretam em uma nova sobrecarga computacional [Ahmed et al. 2015, Ahmed et al. 2016a], usando mensagens extras de controle [Ahmed et al. 2015], ou baseando-se em infraestrutura adicionais [Grassi et al. 2015]. 


\section{A Estratégia EVEN}

EVEN consiste numa estratégia de encaminhamento de interesses para VNDN que faz uso do Tempo de Vida estimado do Enlace (TVE) para determinar se o nó está apto a realizar o encaminhamento de pacotes de interesse. Com isso, evita-se retransmissões desnecessárias na rede. As subseções seguintes descrevem, de forma detalhada, o funcionamento da proposta.

\subsection{Características gerais}

A EVEN é uma estratégia simples uma vez que para seu desenvolvimento e implantação não foram realizadas alterações na estrutura nativa da arquitetura NDN. Além disso, a EVEN não necessita de informações adicionais como sistemas de geo-localização ou adição de novas estruturas de dados para o seu funcionamento. Ao invés disso, é utilizado o TVE para realizar um encaminhamento seletivo e controlar o número de retransmissões. Dessa forma, a proposta foi implementada evitando a adição de complexidade extra. É possível afirmar que a sobrecarga atribuída à EVEN está presente no processo da tomada de decisão e no cálculo do TVE, que possuem uma complexidade de $\Theta(1)$.

Para calcular o TVE é preciso que o nó possua as informações de mobilidade, como velocidade e posição, do nó de origem e do nó atual. Essas informações geralmente são trocadas através de mensagens beacons mas, como já mencionado, tal abordagem acarreta em uso excessivo de recursos da rede e a divulgação de informações desatualizadas. Portanto, para evitar tais problemas, neste trabalho as informações de mobilidade são propagadas através do próprio pacote de interesse o que possibilita coletar informações atuais sobre um determinado nó.

\subsection{Estrutura do pacote de interesse}

A EVEN não exige mudanças na arquitetura NDN padrão. Sendo assim, a estrutura original do pacote de interesse, mostrada na Figura 1, foi mantida.

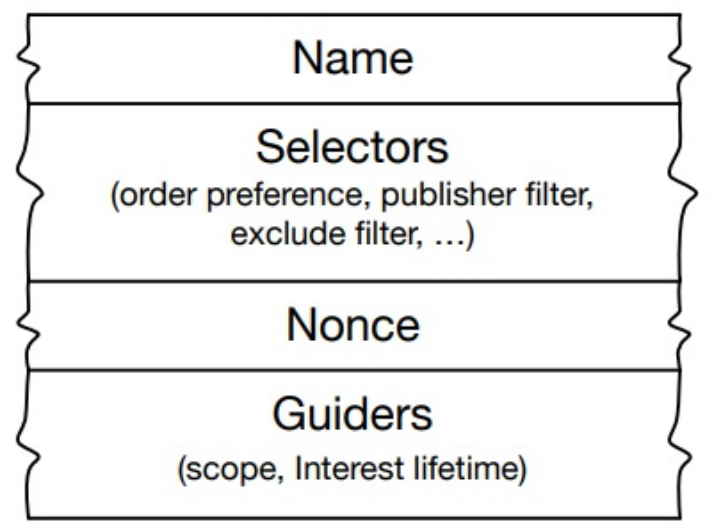

Figura 1. Formato do pacote de interesse [Zhang et al. 2014].

Como é possível observar, os campos do pacote de interesse são:

- Name: representa o nome do interesse, ou seja, o prefixo.

- Selectors: define opções que o pacote pode conter, como tamanho mínimo e máximo de um sufixo, localizadores de chave, filtros, dentre outros. 
- Nonce: consiste em uma string de 4 octetos gerada de forma aleatória que, juntamente com o nome, atua como um identificador único do pacote de interesse.

- Guiders: fornece informações para o processo de encaminhamento como uma lista de possíveis encaminhadores, além de informar o tempo de vida remanescente do interesse.

Para o envio das informações necessárias para o cálculo do TVE é utilizado o campo Name do pacote de interesse, no qual a velocidade e o vetor posição são concatenados ao próprio nome do sufixo do conteúdo solicitado (/Prefixo/Sufixo). Ou seja, supondo que a posição do nó seja -12,9999227,-38,5108559 e sua velocidade igual a $7,9 \mathrm{~m} / \mathrm{s}$, o endereço passado seria uma concatenação do prefixo /rota/UFBA/IME/, com o sufixo -12,9999227,-38,5108559+7.9, formando o endereço /rota/UFBA/IME/12,9999227,-38,5108559+7,9. A partir da adoção desta abordagem, o problema de informações desatualizadas é contornado, pois as informações utilizadas no cálculo do TVE refletem o estado mais recente do nó de origem.

\subsection{Componentes da VNDN}

A Figura 2 apresenta um cenário simplificado em que a estratégia EVEN é adotada. Basicamente, três componentes da VNDN são utilizados, através dos seguintes papéis

- Consumidor $\left(V_{C}\right)$ : consiste em um nó na VANET que está interessado em um determinado conteúdo e irá requisitá-lo através de um pacote de interesse. $V_{C}$ pode atuar tanto como um consumidor como um produtor, no entanto, neste trabalho é considerado apenas um papel por vez.

- Nó intermediário $\left(V_{R}\right)$ : encaminha os pacotes de interesse que são enviados por $V_{C}$ ou por outros nós intermediários. O Nó $V_{R}$ também é responsável por retornar o pacote de dados a $V_{C}$.

- Produtor $\left(V_{P}\right)$ : é o nó que possui os conteúdos solicitados por $V_{C}$. Um nó intermediário também pode atuar como produtor se possuir o conteúdo solicitado em sua Content Store (CS).

É importante destacar que em um ambiente de VNDN todos os nós podem realizar as três funções mencionadas ao mesmo tempo. No entanto, nos experimentos executados os papeis foram distribuídos de forma específica.

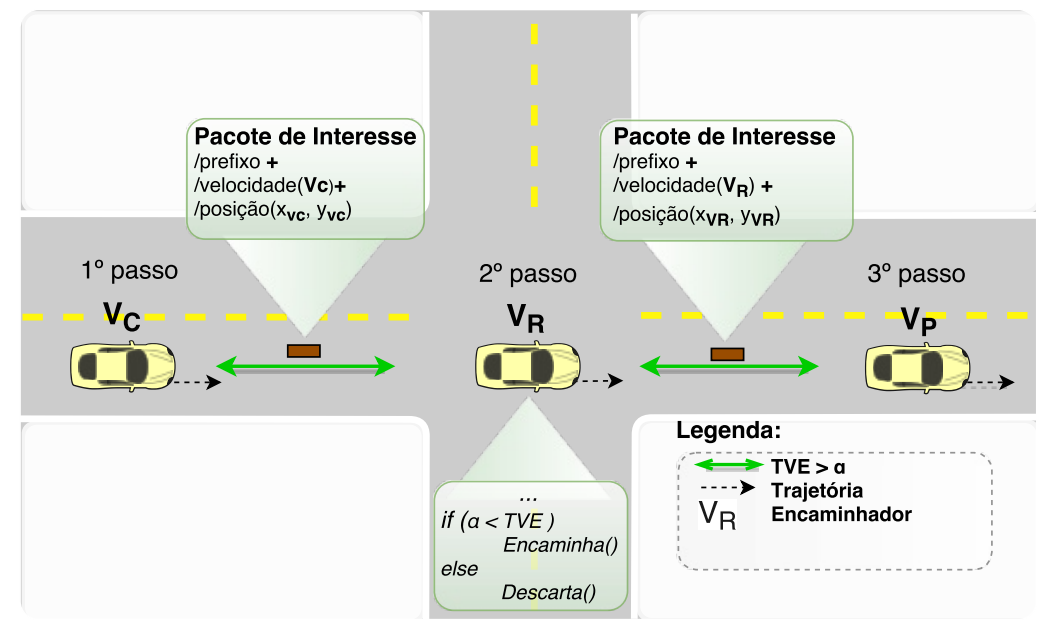

Figura 2. Cenário de VNDN em que a estratégia EVEN é adotada. 
O cenário retratado na Figura 2 ilustra um envio de um interesse, representado pelas setas verdes, originado por $V_{C}$. O nó $V_{C}$ envia o pacote de maneira broadcast para os seu vizinho $V_{R}\left(1^{\circ}\right.$ passo), que por sua vez realiza o processamento do interesse que chega e, caso não possua o conteúdo solicitado, retransmite a mensagem novamente seguindo o mesmo comportamento de $V_{C}$. Tal ação causa um mal uso dos recursos da rede e dos próprios veículos na VNDN nativa, pois todos os nós que não encontrarem o conteúdo em suas CSs irão retransmitir o interesse de maneira broadcast, dessa forma, os nós irão apenas contribuir para o broadcast storm.

\subsection{Tempo de vida do enlace}

No ambiente de VANETs, o gerenciamento da mobilidade é uma tarefa bastante complexa devido a fatores como velocidade e trajetória dos veículos. Diversas abordagens para esse problema podem ser encontradas na literatura, onde, basicamente, são divididas em abordagens proativas e abordagens reativas. Na abordagem proativa, a manutenção das tabelas de roteamento acontecem com ou sem alterações na rede. Já na abordagem reativa a atualização das tabelas é feita somente quando é detectada alguma alteração na rede [Härri et al. 2008].

A abordagem reativa é a mais adequada para o ambiente de VANETs, pois poupa recursos da rede ao realizar a atualização somente quando necessário. No entanto, devido à sua complexidade, a abordagem reativa não é muito explorada. Já o método proativo torna-se inviável pois sobrecarrega a rede ao realizar uma constante inundação de pacotes beacons, causando problemas de atrasos e congestionamentos [Härri et al. 2008, Ahmed et al. 2016a].

Portanto, neste trabalho, assim como em [Härri et al. 2008], é adotado uma técnica reativa para o tratamento da mobilidade e mitigação do broadcast storm, que se baseia no Tempo de Vida estimado do Enlace (TVE). O TVE representa o intervalo de tempo estimado em que um determinado veículo $V_{C}$ irá permanecer dentro do alcance de transmissão de um nó $V_{R}$. Ao utilizar essa métrica, o nó $V_{C}$ pode aferir se realmente será útil no processo de encaminhamento de interesses provenientes do nó $V_{R}$, ou seja, o nó $V_{C}$ realiza uma autoavaliação com base no TVE obtido em relação ao último nó $V_{R}$ que retransmitiu o interesse. A partir do valor obtido, $V_{C}$ analisa se ainda estará na vizinhança de $V_{R}$ quando o pacote de dados com o conteúdo requisitado retornar a $V_{R}$.

Para a realização do cálculo do TVE é necessário que $V_{R}$ possua as informações de velocidade $(v)$, posição $(P)$ de $V_{C}$ e seu próprio alcance de transmissão $(r)$. De posse dessas informações, o nó consegue calcular a distância Euclidiana a partir da Equação 1, que recebe como entrada vetores bidimensionais $x$ e $y$.

$$
d_{P Q}=\sqrt{\left(p_{x}-q_{x}\right)^{2}+\left(p_{y}-q_{y}\right)^{2}}
$$

Dessa forma, pode-se definir $P=\left(p_{x}, p_{y}\right)$ e $Q=\left(q_{x}, q_{y}\right)$. A Equação 2 apresenta uma variação da fórmula do Movimento Retilíneo Uniforme (MRU) para o cálculo do TVE. Seja $r$ o alcance de transmissão de $V_{R}, r$ subtraído a distância atual, $d_{P Q}$, do nó retorna a distância relativa ao limite do raio de atuação de $V_{C}$. Por fim, a predição do tempo é alcançada por meio da divisão da operação $r-d_{P Q}$ pela sua velocidade, Equação 2. 


$$
T V E=\frac{d_{P Q}}{v} \Rightarrow \frac{r-d_{P Q}}{v}
$$

\subsection{Tomada de decisão}

Na EVEN, o processo de encaminhamento se difere das estratégias nativas de NDN, em que um nó ao receber um pacote de interesse e não possuir o conteúdo solicitado irá retransmitir o pacote de maneira broadcast, levando aos problemas destacados anteriormente.

A Figura 2 mostra o funcionamento da EVEN, no qual há um consumidor $V_{C}$ interessado em um conteúdo fornecido pelo produtor $V_{P}\left(1^{\circ}\right.$ passo). Para solicitar o conteúdo o nó envia um pacote de interesse via broadcast para seu vizinho $V_{R}$, que ao receber o interesse calcula o TVE utilizando as informações de mobilidade referentes a $V_{C}$, presentes no prefixo do interesse ( $2^{\circ}$ passo). De posse do TVE, os nós verificam se seu TVE é maior do que um limiar de tempo $\alpha$. O $\alpha$ representa um valor mínimo de TVE em que os nós intermediários se baseiam para determinar se estão aptos a satisfazer o interesse. Após calcular o TVE, $V_{R}$ avalia que o mesmo é superior a $\alpha$, sendo assim, $V_{R}$ decide atuar como um encaminhador, mas antes de enviar o interesse as informações de mobilidade presentes no mesmo são substituídas pelas próprias informações de $V_{R}$. Logo em seguida, o interesse é encaminhado para o produtor que satisfaz a requisição ( $3^{\circ}$ passo).

Vale destacar que o valor de $\alpha$ pode variar para cada veículo e pode ser determinado utilizando métricas como tempo de atraso médio da rede, distância, TVE médio, etc. No entanto, para fins de simplicidade, neste trabalho foi adotado um $\alpha$ fixo para todos os nós, que assume os valores de 5 e 10 segundos.

O Algoritmo 1 detalha o processo da tomada de decisão realizado pelo veículo $V_{R}$ na Figura 2. Nas linhas 2-3 é verificado se o conteúdo requisitado não se encontra na CS e se não há entrada na PIT para o nome do interesse, respectivamente. Caso o conteúdo esteja na CS, então o mesmo será enviado de volta ao consumidor (linhas 20-21). No caso de uma entrada correspondente ao nome do interesse for encontrada na PIT, será adicionada a interface de entrada do interesse e o mesmo será descartado (linhas 16-17). Na linha 9 é calculado o TVE com a função calculaTVE que recebe como parâmetro os vetores de velocidade e posição do nó de origem, ou seja, o nó que propagou o interesse, além das informações de mobilidade e alcance de transmissão do nó atual.

A tomada de decisão de encaminhamento ocorre na linha 10, em que é verificado se o TVE é maior do que $\alpha$. Se sim, então o pacote será encaminhado com as informações de mobilidade substituídas pelas informações de mobilidade referentes ao nó atual. Caso contrário, o pacote de interesse será descartado pois o nó avalia que ele não está apto a retornar o conteúdo para o consumidor.

\section{Experimentação}

Esta Seção apresenta quais cenários, parâmetros, métricas e fatores utilizados na avaliação da EVEN. A Subseção 4.2 introduz as ferramentas e comportamento das simulações. As métricas de desempenho utilizadas são detalhadas na Subseção 4.3. Por fim, a Subseção 5 conclui com a análise dos resultados. 


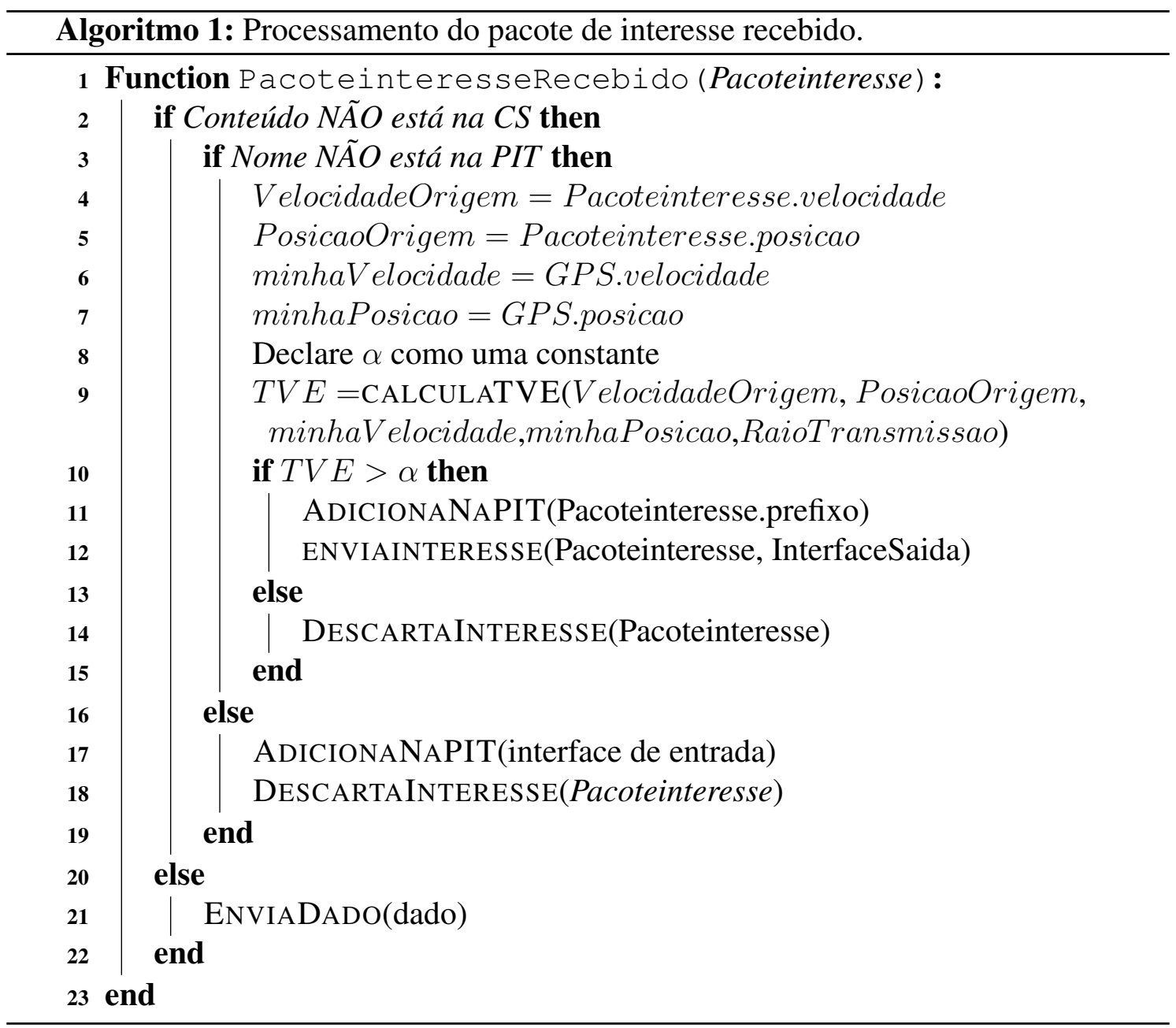

\subsection{Ambiente de simulação}

Para as simulações foi utilizado o simulador de redes NS-3 $3^{1}$, que implementa o padrão IEEE 802.11p para redes veiculares. No entanto, o NS-3 por si só não provê suporte a NDN, portanto, para o desenvolvimento da proposta foi adotado o módulo ndnSIM [Mastorakis et al. 2015], que fornece uma implementação completa da pilha de protocolos NDN além de APIs (Application Programming Interfaces) que facilitam a criação de novas aplicações.

Os cenários veiculares escolhidos foram os cenários de rodovia e urbano, ambos representados por traces de mobilidade gerados pelo $\mathrm{SUMO}^{2}$ (Simulation of Urban MObility). O cenário urbano é apresentado como uma área e $1.6 \times 1.5 \mathrm{Km}$, feito de grids, com duas avenidas horizontais e quatro avenidas verticais e cada avenida contendo duas faixas e tráfego veicular fluindo nos dois sentidos. Já o cenário de rodovia consiste em uma pista de $1 \mathrm{Km}$ de comprimento com duas faixas nos dois sentidos.

\footnotetext{
${ }^{1}$ https://www.nsnam.org/

${ }^{2}$ http://sumo.dlr.de/index.html
} 


\subsection{Metodologia}

As simulações ocorreram da seguinte forma. Em cada execução foi escolhido um produtor fixo e a cada rodada um novo veículo assumiu o papel de consumidor, de modo a permitir que todos os veículos, exceto o produtor, atuassem como consumidor uma vez. É importante ressaltar que os experimentos executados seguiram as mesmas especificações para cada estratégia comparada, ou seja, o ambiente de simulação utilizado na EVEN foi o mesmo usado no Multicast Nativo e Best-Route Nativo. Para tornar o cenário mais realista as requisições foram iniciadas somente após um período de tempo depois do início da simulação, para permitir que os veículos se espalhassem pelo cenário [Ahmed et al. 2016a]. A Tabela 1, sumariza os parâmetros da simulação.

Tabela 1. Parâmetros da Simulação.

\begin{tabular}{ll}
\hline Parâmetro & Valor \\
\hline Simulador de redes & ndnSIM \\
Modelo de rádio propagação & Nakagami \\
Número de nós & $25,50,75$ e 100 \\
Tempo de simulação & 150 segundos \\
Cenários de mobilidade & Urbano e Rodovia \\
Alcance de transmissão & 200 metros \\
Protocolo de enlace & $802.11 \mathrm{p}$ \\
Tamanho do pacote de dados & $1024 \mathrm{~B}$ \\
Política de cache & Sem cache \\
Quantidade máxima de conteúdos requisitados & 1000 \\
Valor de $\alpha$ & 5 e 10 segundos \\
Cálculo do tempo de vida do enlace & baseado em [Härri et al. 2008] \\
Estratégias de encaminhamento comparadas & EVEN, Best-Route Nativo e Multicast Nativo \\
\hline
\end{tabular}

Como apresentado na Tabela 1, as estratégias utilizadas para comparação foram Best-Route Nativo e Multicast Nativo. Não foram realizadas comparações com as estratégias apresentadas na Seção 2 pois estas necessitam de apoio de infraestrutura nas proximidades (do inglês, Vehicle-to-Infrastructure - V2I), enquanto, a estratégia proposta neste trabalho é exclusivamente V2V, ou seja, não requer a presença de RSUs para o seu funcionamento. Portanto, tal comparação se tornaria complexa e tendenciosa.

\subsection{Métricas de desempenho}

A fim de analisar o desempenho da proposta e verificar se objetivo principal foi alcançado, foram estabelecidas variáveis de resposta para a validação da proposta, tais como:

- Taxa de satisfação de interesses (Interest Satisfaction Rate- ISR): descreve a porcentagem de pacotes de interesse satisfeitos durante a simulação. Através dessa métrica, é possível avaliar a eficiência da EVEN em relação a entrega dos conteúdos solicitados pelo consumidor. Essa métrica auxilia na análise os efeitos colaterais da proposta, uma vez que o número de interesses propagados é reduzido haverá menos encaminhadores para retornar o conteúdo ao consumidor, portanto, ocorrerá queda de desempenho no quesito confiabilidade.

- Pacotes de interesse encaminhados (Forwarded Interest Packets - FIP): representa o número médio de pacotes de interesse retransmitidos pelos nós ao longo da 
simulação. Essa métrica é de significativa importância pois reflete o principal objetivo da EVEN, que é a redução no número de retransmissões de pacotes de dados.

- Pacotes de dados encaminhados (Forwarded Data Packets - FDP): representa o número médio de retransmissão de pacotes de dados. Com a redução do FIP, é esperado que o número de dados retornados ao consumidor também diminua, pois menos nós irão retransmitir os interesses. A redução desse número pode impactar em um melhor funcionamento da rede, uma vez que os pacotes de dados possuem carga útil e, portanto, podem saturar os enlaces de forma mais rápida [Ahmed et al. 2016a].

- Atraso na satisfação de interesses (Interest Satisfaction Delay - ISD): especifica a duração de tempo, em segundos, entre o envio de uma requisição efetuada por um consumidor e a chegada do dado referente a essa requisição. Basicamente, essa métrica representa o atraso médio para consumir um determinado conteúdo da rede. O ISD reflete o grau de satisfação dos usuários.

\section{Resultados e análises}

Nesta Seção são apresentados e discutidos os resultados obtidos a partir das simulações realizadas no ndnSIM.

\subsection{Cenário de rodovia}

Na Figura 3 é apresentado os resultados relacionados a execução da EVEN em cenário de rodovia. Observando a Figura 3(a), pode-se inferir que a estratégia Best-Route Nativo conseguiu superar as demais ao manter o FDP abaixo de 4000 retransmissões por nó. Por outro lado, se for observado seu ISR (Figura 3(b)) percebe-se que a estratégia falhou no quesito confiabilidade, em que conseguiu satisfazer somente cerca de $4 \%$ dos interesses requisitados, ou seja, o FDP manteve-se baixo pois a estratégia falhou em encontrar a melhor rota para o produtor. Portanto, a estratégia com a melhor performance na redução do FDP, levando em consideração o seu ISR, é a EVEN com um $\alpha$ igual à 10. Além disso, é importante observar que no cenário com 100 veículos há um número maior de retransmissões mesmo com a EVEN, devido a maior proximidade entre os nós que elevam o TVE.

A confiabilidade é retratada na Figura 3(b), onde é possível notar que a estratégia Multicast Nativo possui a maior taxa de interesses satisfeitos, mas se for observado seu FDP e seu FIP (Figura 3(c)) torna-se evidente que essa estratégia não previne o broadcast storm. Já a EVEN com $\alpha$ igual à 5 e 10 manteve um ISR semelhante ao alcançado pela estratégia Multicast Nativo, em contrapartida, o seu FDP e FIP foram melhores se comparado às demais estratégias. Esse comportamento representa um tradeoff, ou seja, por um lado há perda de desempenho no quesito confiabilidade mas, por outro lado, a EVEN utiliza menos recursos da rede para atender a uma quantidade semelhante de requisições.

A Figura 3(c) mostra o FIP obtido no ambiente de rodovia, onde a capacidade da EVEN ao reduzir o número de retransmissões se mostra superior as demais. Além disso, é possível notar que a EVEN, com um $\alpha$ igual a 10, obteve um FIP ainda menor, com uma diferença média de $\approx 35 \%$ em comparação a estratégia Multicast Nativo. Isso ocorre porque à medida que o $\alpha$ cresce, a filtragem dos nós torna-se mais criteriosa, ou seja, apenas os nós com um TVE elevado são considerados aptos a encaminhar os interesses. 


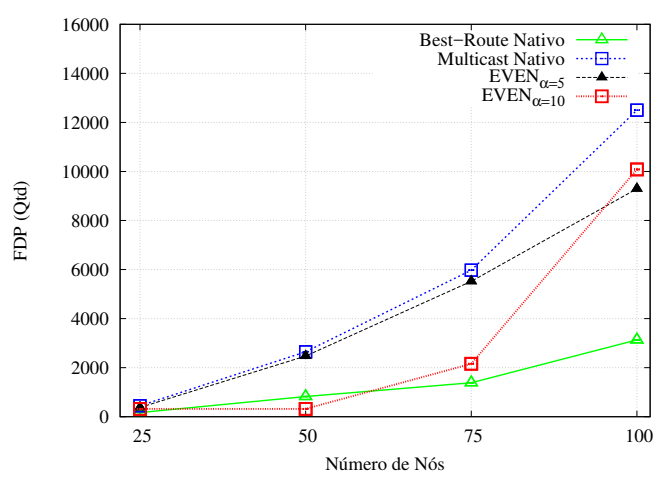

(a) FDP.

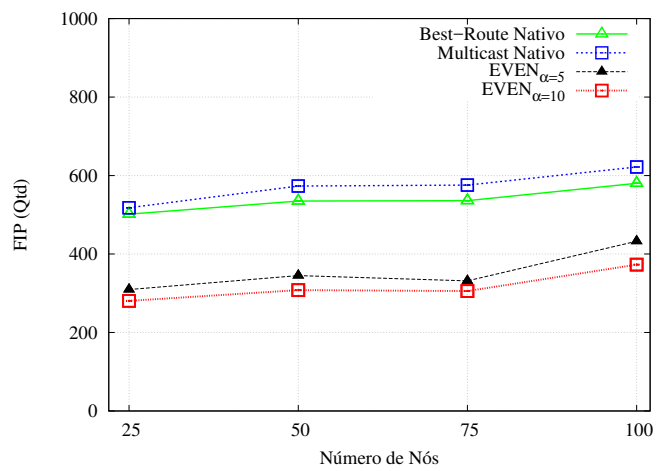

(c) FIP.

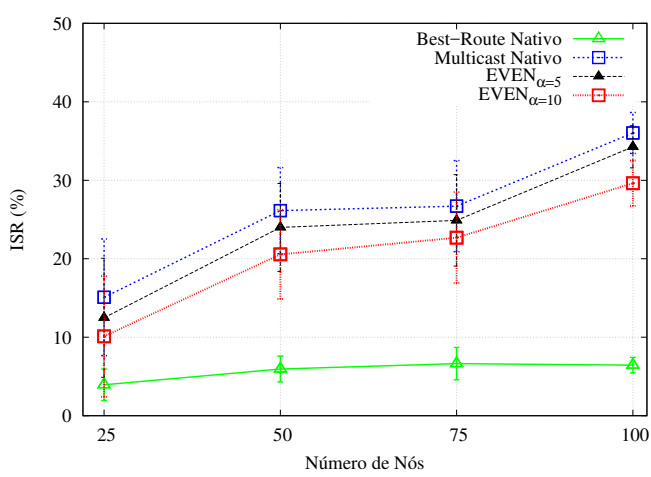

(b) ISR.

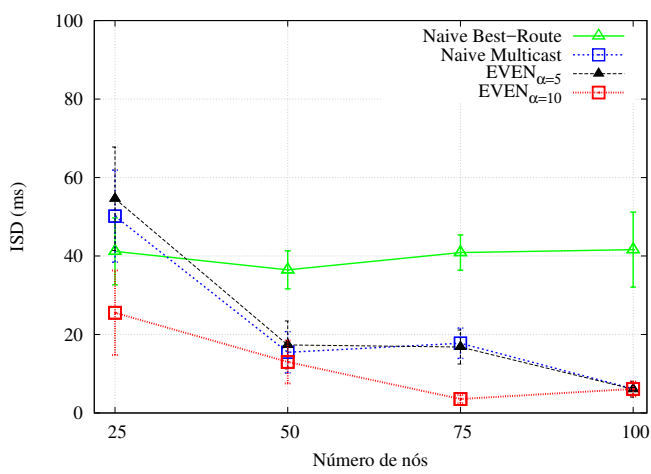

(d) ISD.

Figura 3. Avaliação da EVEN em cenário de rodovia.

Apesar de obter um ISR inferior ao obtido pela estratégia Multicast Nativo, a EVEN alcançou uma redução considerável no tempo de resposta necessário para satisfazer os interesses, como mostrado na Figura 3(d). A redução do ISD demonstra que a EVEN consegue criar links de comunicação estáveis entre os nós, o que permite um retorno seguro dos dados. A Tabela 2 destaca de forma resumida os ganhos $(+)$ e perdas (-) da EVEN comparado a estratégia Multicast Nativo em um cenário de rodovia.

Tabela 2. Síntese do desempenho da EVEN comparado a estratégia Multicast Nativo em cenário de rodovia.

\begin{tabular}{|l|l|l|l|l|}
\cline { 2 - 5 } \multicolumn{1}{c|}{} & FIP & FDP & ISR & ISD \\
\hline EVEN $_{\alpha=5}$ & $+44,76 \%$ & $+12,66 \%$ & $-3 \%$ & $-3,55 \%$ \\
\hline EVEN $_{\alpha=10}$ & $+49,78 \%$ & $+31,54 \%$ & $-6 \%$ & $+36,39 \%$ \\
\hline
\end{tabular}

A partir dos valores presentes na Tabela 2 pode ser comprovado que os ganhos atribuídos a redução do FIP, FDP e ISD superam as perdas relacionadas ao ISR.

\subsection{Cenário urbano}

Os resultados das simulações realizadas no ambiente urbano estão representados na Figura 4. Nesse ambiente a complexidade envolvida é maior se comparado ao cenário de rodovia, devido a fatores como mudança de sentido, velocidade, presença de interseções 
e semáforos. Tais características criam situações variadas, como em casos onde o consumidor pode estar na vizinhança do produtor e alcança um ISR elevado, já em outros, o consumidor pode estar localizado em uma área fora do alcance de qualquer outro nó e, portanto, terá um ISR próximo ou igual a $0 \%$. Esse comportamento pode ser evidenciado nas variações dos resultados obtidos.

Como ilustrado na Figura 4(a), a EVEN reduziu o FDP em todos os casos, se comparado à estratégia Multicast Nativo, onde a maior diferença encontra-se no cenário com 50 nós. É importante mencionar que apesar da estratégia Best-Route Nativo apresentar um FDP abaixo das demais, a partir de seu ISR é possível inferir que o mesmo apresenta-se inferior às outras estratégias, ou seja, o Best-Route Nativo não consegue sequer alcançar o produtor, portanto, há poucos pacotes de dados retornados ao consumidor.

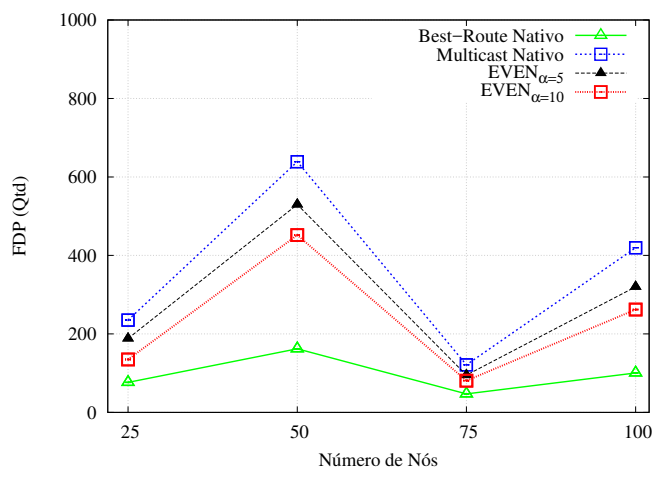

(a) FDP.

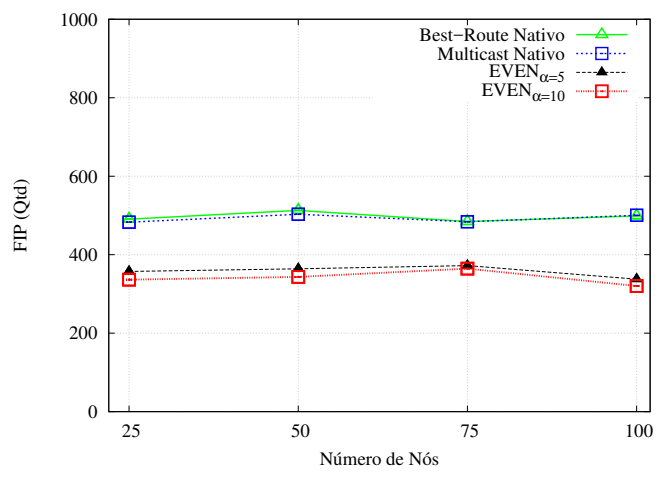

(c) FIP.

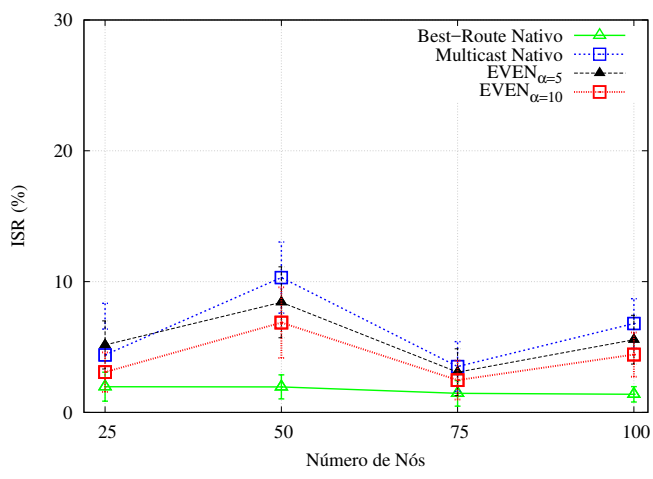

(b) ISR.

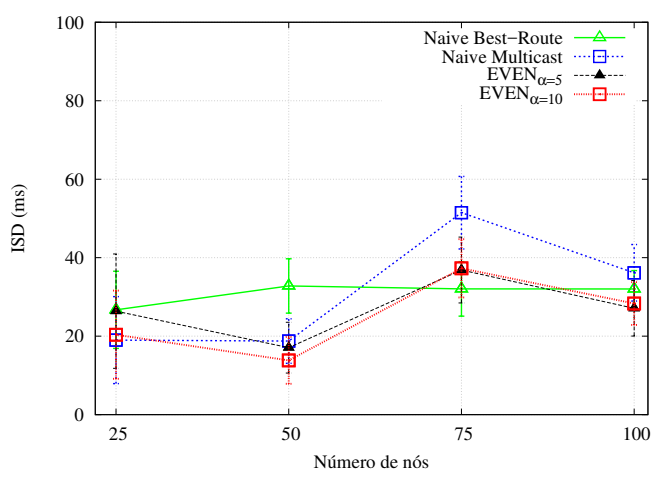

(d) ISD.

Figura 4. Avaliação da EVEN em cenário urbano.

A Figura 4(b) mostra que no cenário com 25 nós a EVEN supera o ISR alcançado pelas demais estratégias. Entretanto, nota-se que houve uma queda no ISR à medida que o número de nós aumenta. Novamente, esse comportamento pode ser compreendido como um sacrifício tolerável em relação aos resultados obtidos com o FIP, FDP e ISD. A EVEN com um $\alpha$ igual a 10 conseguiu superar às demais estratégias, uma vez que os nós escolhidos como encaminhadores possuem enlaces com um tempo de vida alto e conseguiram retornar o pacote de dados de forma mais rápida ao mesmo tempo em que reduziu o número de retransmissões.

A EVEN, além de reduzir o FDP, manteve um ISR semelhante ao obtido pela estratégia Multicast Nativo e alcançou o objetivo principal que consiste na redução do 
FIP, Figura 4(c), em que manteve-se abaixo das demais estratégias em todos os cenários. Mesmo em um ambiente imprevisível, a EVEN diminuiu o número de retransmissões sem grandes impactos em seu desempenho ao satisfazer os interesses.

Por fim, a Figura 4(d) mostra que a EVEN conseguiu reduzir o ISD em torno de $\approx 5 \%$ com um $\alpha$ igual a 5 e em $\approx 17 \%$ com $\alpha$ igual a 10 . A redução do ISD é importante em VNDN uma vez que mensagens de alerta devem ser entregues ao consumidor de forma rápida para prevenir possíveis acidentes. A estratégia Best-Route Nativo, inicialmente, se mostra superior a EVEN, mas se for observado seu ISR nota-se que tal afirmação é inválida. A Tabela 3 evidencia que os ganhos da EVEN, comparado a estratégia Multicast Nativo, no quesito redução de retransmissões e tempo de resposta superam a queda no ISR mesmo em um cenário alta.

\section{Tabela 3. Síntese do desempenho da EVEN comparado a estratégia Multicast Nativo em cenário urbano.}

\begin{tabular}{|l|l|l|l|l|}
\cline { 2 - 5 } \multicolumn{1}{c|}{} & FIP & FDP & ISR & ISD \\
\hline EVEN $_{\alpha=5}$ & $+30,47 \%$ & $+34,30 \%$ & $-2 \%$ & $+5,87 \%$ \\
\hline EVEN $_{\alpha=10}$ & $+34,41 \%$ & $+43,35 \%$ & $-3,9 \%$ & $+17,05 \%$ \\
\hline
\end{tabular}

\section{Conclusão e trabalhos futuros}

A gerência da mobilidade em VANETs é uma tarefa complexa e apesar da união com as Redes de Dados Nomeados, que apresentam uma gama de caraterísticas que se adaptam de forma eficiente ao ambiente de VANETs, ainda existem diversos desafios em aberto que precisam serem resolvidos para alcançar um funcionamento adequado. Um desses desafios é o broadcast storm de pacotes de interesse que acarretam em uso desnecessário de recursos dos veículos e causam atrasos na rede. Para amenizar os efeitos desse problema foi proposto neste trabalho uma técnica de encaminhamento de interesses que se baseia no Tempo de Vida Estimado do enlace para realizar a decisão de encaminhamento. Pelos resultados obtidos foi possível inferir que a proposta alcançou os objetivos esperados, onde reduziu de forma eficiente o número de pacotes de interesse retransmitidos e, consequentemente, diminuiu a quantidade de réplicas dos pacotes de Dados retornados ao consumidor. Além disso, a experiência do usuário foi mantida estável, sendo que a taxa de satisfação de interesses não sofreu grandes perdas.

Para trabalhos futuros, a tomada de decisão será aprimorada para os ambientes de rodovia. O valor do $\alpha$ será determinado de maneira dinâmica levando em consideração critérios como tempo de vida médio do enlace, atraso médio, distância mínima, etc. Por fim, a proposta será avaliada em ambiente mais realista com uma variação maior no número de nós e comparada com a estratégia CODIE [Ahmed et al. 2016b].

\section{Agradecimentos}

Os autores agradecem o apoio do Conselho Nacional de Desenvolvimento Científico e Tecnológico (CNPq) e da Fundação de Amparo à Pesquisa do Estado da Bahia (FAPESB).

\section{Referências}

Ahmed, S. H., Bouk, S. H., and Kim, D. (2015). RUFS: RobUst forwarder selection in vehicular content-centric networks. IEEE Communications Letters, 19(9):1616-1619. 
Ahmed, S. H., Bouk, S. H., Yaqub, M. A., Kim, D., and Gerla, M. (2016a). Conet: Controlled data packets propagation in vehicular named data networks. In Consumer Communications \& Networking Conference (CCNC), 2016 13th IEEE Annual, pages 620-625. IEEE.

Ahmed, S. H., Bouk, S. H., Yaqub, M. A., Kim, D., Song, H., and Lloret, J. (2016b). CODIE: Controlled data and interest evaluation in vehicular named data networks. IEEE Transactions on Vehicular Technology, 65(6):3954-3963.

Grassi, G., Pesavento, D., Pau, G., Zhang, L., and Fdida, S. (2015). Navigo: Interest forwarding by geolocations in vehicular named data networking. In World of Wireless, Mobile and Multimedia Networks (WoWMoM), 2015 IEEE 16th International Symposium on a, pages 1-10. IEEE.

Härri, J., Bonnet, C., and Filali, F. (2008). Kinetic mobility management applied to vehicular ad hoc network protocols. Computer Communications, 31(12):2907-2924.

Karagiannis, G., Altintas, O., Ekici, E., Heijenk, G., Jarupan, B., Lin, K., and Weil, T. (2011). Vehicular networking: A survey and tutorial on requirements, architectures, challenges, standards and solutions. IEEE communications surveys \& tutorials, 13(4):584-616.

Kuai, M., Hong, X., and Yu, Q. (2016). Density-aware delay-tolerant interest forwarding in vehicular named data networking. In Vehicular Technology Conference (VTC-Fall), 2016 IEEE 84th, pages 1-5. IEEE.

Majeed, M. F., Ahmed, S. H., and Dailey, M. N. (2017). Enabling push-based critical data forwarding in vehicular named data networks. IEEE Communications Letters, 21(4):873-876.

Maryam, H., Wahid, A., and Shah, M. A. (2017). Mitigating broadcast storm in interest/data packet forwarding in vehicular content centric networking. In 2017 International Conference on Communication Technologies (ComTech), pages 162-167.

Mastorakis, S., Afanasyev, A., Moiseenko, I., and Zhang, L. (2015). ndnsim 2.0: A new version of the ndn simulator for ns-3. NDN, Technical Report NDN-0028.

Saxena, D., Raychoudhury, V., Suri, N., Becker, C., and Cao, J. (2016). Named data networking: a survey. Computer Science Review, 19:15-55.

Yaqub, M. A., Ahmed, S. H., Bouk, S. H., and Kim, D. (2016). Interest forwarding in vehicular information centric networks: a survey. In Proceedings of the 31st Annual ACM Symposium on Applied Computing, pages 724-729. ACM.

Yu, Y.-T., Dilmaghani, R. B., Calo, S., Sanadidi, M., and Gerla, M. (2013). Interest propagation in named data manets. In Computing, Networking and Communications (ICNC), 2013 International Conference on, pages 1118-1122. IEEE.

Zhang, L., Afanasyev, A., Burke, J., Jacobson, V., Crowley, P., Papadopoulos, C., Wang, L., Zhang, B., et al. (2014). Named data networking. ACM SIGCOMM Computer Communication Review, 44(3):66-73. 